\title{
Ventricular aneurysm in a chronic Chagas disease patient from the Brazilian Amazon region
}

\author{
Aneurisma ventricular em paciente chagásico crônico \\ da Amazônia brasileira
}

João Marcos Bemfica Barbosa Ferreira ${ }^{1}$, Jorge Augusto de Oliveira Guerra ${ }^{2,3}$ and Maria das Graças Vale Barbosa ${ }^{2,3}$

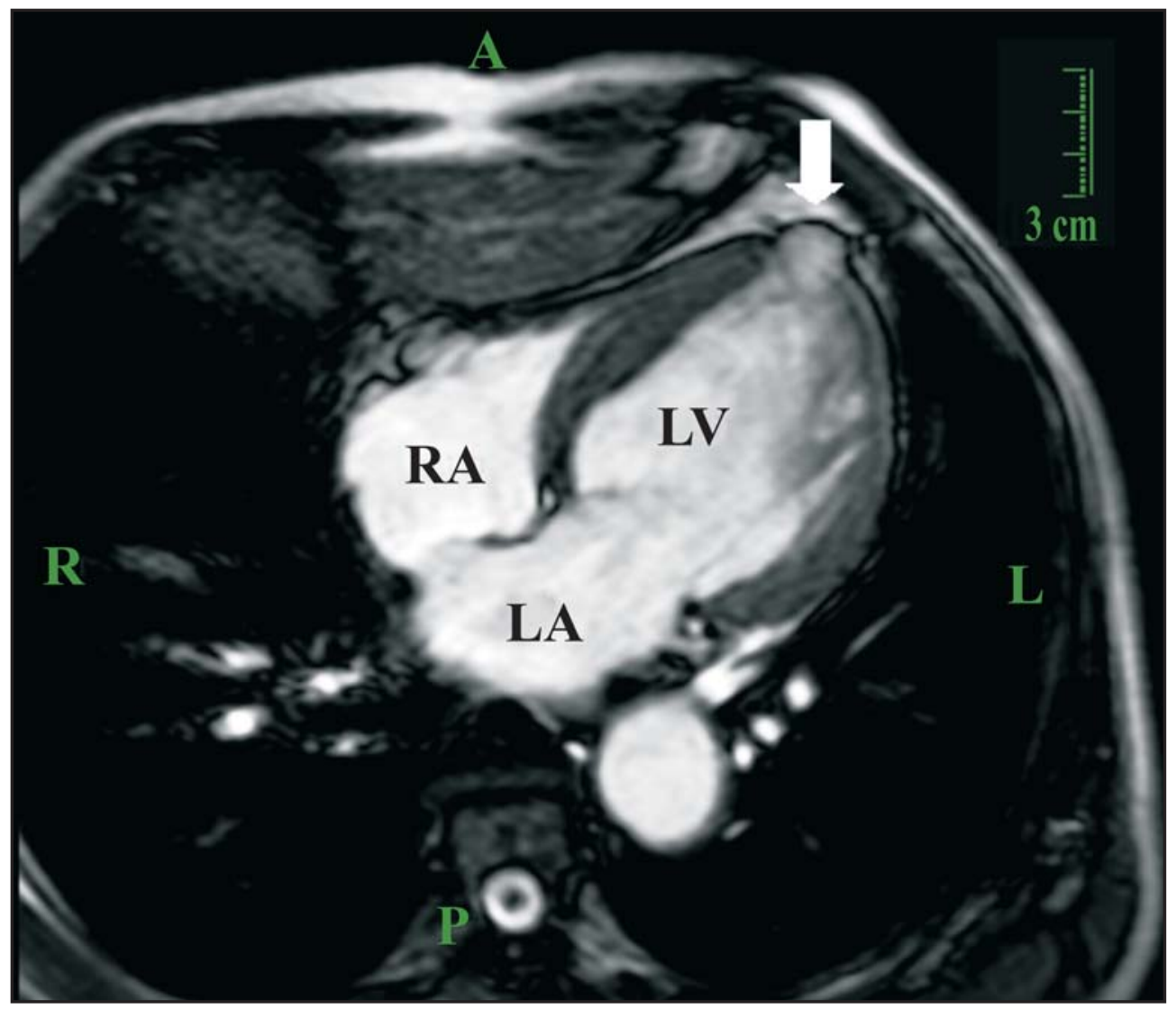

$\mathbf{A}$

\footnotetext{
1. Francisca Mendes University Hospital, Federal University of Amazonas, Manaus, AM, Brazil. 2. Foundation of Tropical Medicine of Amazonas, Manaus, AM, Brazil. 3. University of Amazonas State, Manaus, AM, Brazil.

Address to: Dr. João Marcos Bemfica Barbosa Ferreira. Rua Ramos Ferreira 199/1501, Aparecida, 69010-120 Manaus, AM, Brazil.

e-mail: jmbemfica@hotmail.com

Tel: $55923232-5683$

Received in 20/03/2009

Accepted in 14/05/2009
} 
The patient was a 69-year-old man from Manacapuru, State of Amazonas, who was living in Manaus, Amazonas. Between the ages of 30 and 33 years, he worked extracting fiber from piassaba palm trees in the Aracá river region of the municipality of Barcelos, Amazonas. Three years ago, he started presenting dyspnea upon physical exertion, orthopnea and paroxysmal nocturnal dyspnea. He underwent an electrocardiographic examination that showed a pattern of left ventricular overload. An echocardiogram showed a left ventricular diastolic diameter of $73 \mathrm{~mm}$, left ventricular ejection fraction of $28 \%$, inferior wall akinesia and an image suggesting left ventricular apical aneurysm. He underwent cardiac magnetic resonance imaging, which confirmed the image of apical aneurysm of glove finger type (Figure A). He underwent a cardiac catheterization, which did not show any coronary artery obstructions. Serological tests for Chagas disease (indirect immunofluorescence and ELISA with recombinant antigen) gave positive reactions. The diagnosis was confirmed through a strongly reactive Western blot test (TESA blot). The prevalence of ventricular aneurysms in Chagas disease patients varies according to the diagnostic method used and the geographical region studied. The frequency of aneurysms diagnosed by means of echocardiography ranges from 14 to $46 \%$. In the Amazon region, only five cases of chronic Chagas disease myocardiopathy have so far been described in autochthonous patients. Of these, three presented apical aneurysm of glove finger type, which is typical of Chagas disease involvement. The patient described here is a further case of autochthonous chronic Chagas myocardiopathy from the Amazon region with an image of aneurysm that is typical of Trypanosoma cruzi infection. Therefore, it is important to consider Chagas disease as a differential diagnosis for cardiopathy in the Amazon region.

0 paciente, de 69 anos, é natural de Manacapuru, AM e procedente de Manaus, AM. Trabalhou dos 30 aos 33 anos na extração de fibras de piaçaba no Rio Aracá, município de Barcelos, AM. Há três anos iniciou quadro de dispnéia aos esforços, ortopnéia e dispnéia paroxística noturna. Tem eletrocardiograma com padrão de sobrecarga ventricular esquerda. 0 ecocardiograma demonstrou diâmetro diastólico do ventrículo esquerdo de $73 \mathrm{~mm}$, fração de ejeção ventricular esquerda de $28 \%$, acinesia da parede inferior e imagem sugestiva de aneurisma apical ventricular esquerdo. Submeteu-se a ressonância magnética cardíaca que confirmou a imagem de aneurisma apical tipo dedo de luva (Figura A). Foi submetido a cateterismo cardíaco que demonstrou ausência de obstruções coronarianas. A sorologia para doença de Chagas resultou reagente na imunofluorescência indireta e no método ELISA com antígeno recombinante. 0 diagnóstico foi confirmado com Western-Blot (TESA-Blot) fortemente reagente. A prevalência dos aneurismas ventriculares em pacientes chagásicos varia de acordo com o método diagnóstico utilizado e a região geográfica estudada. A frequência de aneurismas diagnosticados por ecocardiograma varia de 14 a 46\%. Na Amazônia, foram descritos, até o momento, apenas cinco casos de miocardiopatia chagásica crônica em pacientes autóctones. Destes, três apresentavam aneurisma apical do tipo dedo de luva, típico de acometimento pela doença de Chagas. 0 paciente descrito é mais um caso de miocardiopatia chagásica crônica autóctone da Amazônia com imagem de aneurisma típico de infecção pelo Trypanosoma cruzi. Portanto, é importante considerar a doença de Chagas no diagnóstico diferencial das cardiopatias da região amazônica.

\section{REFERENCES}

1. Albajar PV, Laredo SV, Terrazas MB, Coura JR. Miocardiopatia dilatada em pacientes com infecção chagásica crônica. Relato de dois casos fatais autóctones do Rio Negro, Estado do Amazonas. Revista da Sociedade Brasileira de Medicina Tropical 36: 401-407, 2003.

2. Borges-Pereira J, Xavier SS, Sousa AS, Castro JAF, Zauza PL, Coura JR. Prevalência de aneurismas do ventrículo esquerdo em pacientes chagásicos crônicos de duas áreas do Estado do Piauí. Revista da Sociedade Brasileira de Medicina Tropical 40: 521-526, 2007.

3. Xavier SS, Sousa AS, Albajar PV, Junqueira ACV, Bóia MN, Coura JR. Cardiopatia chagásica crônica no Rio Negro, Estado do Amazonas. Relato de três novos casos autóctones, comprovados por exames sorológicos, clínicos, radiográficos do tórax, eletro e ecocardiográficos. Revista da Sociedade Brasileira de Medicina Tropical 39: 211-216, 2006. 